\title{
Effect of curcumin-lipiodol emulsion combined with absorbable anti-adhesion film through double embolic pathway on angiogenesis and overall survival (OS) time of VX2 liver cancer rabbits
}

\author{
Chuanyu Yang, Zheng Xu, Kai Nie, Jing Liu \\ Department of General Surgery, Affiliated Southeast Hospital of Xiamen University, Zhangzhou 363000, China \\ Contributions: (I) Conception and design: J Liu; (II) Administrative support: Z Xu; (III) Provision of study materials or patients: K Nie; (IV) Collection \\ and assembly of data: Z Xu; (V) Data analysis and interpretation: C Yang; (VI) Manuscript writing: All authors; (VII) Final approval of manuscript: \\ All authors. \\ Correspondence to: Jing Liu. Department of General Surgery, Affiliated Southeast Hospital of Xiamen University, No. 269, Zhanghua Middle Road, \\ Zhangzhou 363000, China. Email: liujdoctor@hotmail.com.
}

Background: Curcumin is a promising anti-tumor drug in recent years. The main function of absorbable anti-adhesion film is to prevent adhesions after surgical operation. The effects of curcumin-lipiodol emulsion injected via the hepatic artery and portal vein catheter combined with using absorbable anti-adhesion film around the liver angiogenesis are unknown. This article mainly discusses the effects of these combined strategies on the inhibition or reduction of postoperative angiogenesis and prolonging survival time of rabbit model.

Methods: Forty model rabbits with VX2 liver cancer were divided into four groups. Group A is the control group and its intervention is a simple lipiodol emulsion group. The intervention in group B was a simple lipiodol emulsion combined with an anti-adhesion membrane. The intervention in group $\mathrm{C}$ was a mixture of curcumin and lipiodol. The intervention in group D was a mixed emulsion of curcumin and lipiodol combined with an anti-adhesion membrane. The levels of aspartate aminotransferase (ALT) and alanine aminotransferase (AST) in the model rabbits were compared to analyze the damage of liver cells 1 day before embolization and 3, 7, 14 days after the embolization. In addition, the levels of vascular endothelial growth factor (VEGF) and microvessel density (MVD) in residual cancer tissues were determined by immunohistochemistry and Western blot. Finally, overall survival time (OS) of each group was recorded.

Results: The results showed that the ALT and AST levels of venous blood before and after embolization in each group of rabbits were not changed significantly $(\mathrm{P}>0.05)$. The levels of VEGF and MVD in the group $\mathrm{C}$ and group $\mathrm{D}$ were lower than those in the group $\mathrm{A}(\mathrm{P}<0.05)$. However, there was no significant difference between group B and group A $(\mathrm{P}>0.05)$. The decrease of VEGF and MVD in group D was most obvious, and group $\mathrm{D}$ had a significant difference compared with group $\mathrm{C}(\mathrm{P}<0.05)$. Western blot analysis showed that the protein expression of VEGF was consistent with immunohistochemical staining. The overall survival (OS) time of group B showed no significant difference compared with group A $(\mathrm{P}>0.05)$. The OS time of group $\mathrm{C}$ and group $\mathrm{D}$ was longer than that of group $\mathrm{A}(\mathrm{P}<0.05)$. Group $\mathrm{D}$ showed significantly difference on prolongation of the survival time than group $\mathrm{C}(\mathrm{P}<0.05)$.

Conclusions: Overall, the curcumin-lipiodol emulsion injected into the portal vein and hepatic artery supplying the tumor can significantly inhibit angiogenesis and play a role in prolonging the OS time of model rabbits. The application of anti-adhesion film alone cannot inhibit tumor angiogenesis and play a role in prolonging the OS time of model rabbits. However, curcumin-lipiodol emulsion combined with antiadhesion film can obviously promote these effects.

Keywords: Curcumin-lipiodol emulsion; rabbit VX2 liver cancer model; vascular endothelial growth factor (VEGF); microvessel density (MVD); overall survival (OS); neovascularization 
Submitted Apr 14, 2019. Accepted for publication Oct 25, 2019.

doi: 10.21037/tcr.2019.12.15

View this article at: http://dx.doi.org/10.21037/tcr.2019.12.15

\section{Introduction}

Hepatocellular carcinoma (HCC) is one of the most common malignant tumors in the world. China is a high incidence area of liver cancer. The latest statistics show that there are 782,000 new cases of liver cancer and 745,000 death each year in the world. However, new cases and deaths in China account for approximately $50 \%$ of the total cases and death (1). Surgical resection is the first choice for the treatment of early-diagnosed HCC, but the majority of patients in the clinic have reached the advanced stage when diagnose. Most of the patients are accompanied by hepatitis $\mathrm{B}$ and cirrhosis. The patients who really conform to surgical resection indications are less than $25 \%$ (2). Transcatheter arterial chemoembolization (TACE) is a non-curative treatment for patients with advanced HCC who cannot be surgically resection. Long term follow-up study showed that the short-term efficacy of TACE was better, but the recurrence rate was high after operation and the long-term effect was not satisfactory (3). Curcumin is one of the main effective components of Curcuma longa, which can reduce the occurrence of the tumor. It can inhibit the proliferation of gastric cancer, lung cancer, leukemia and other tumor cells (4-6). Curcumin has a significant anti-angiogenic effect and it has become a hot spot of anti-tumor research at home and abroad $(7,8)$. The absorbable anti-adhesion film is mainly used to prevent tissue adhesion after surgery (9). We hypothesized that the absorbable anti-adhesive membrane can block or delay the formation of peripheral blood vessels of the liver, thereby enhancing the therapeutic effect of liver cancer and prolonging the overall survival time of the animal. Therefore, based on the blockade of hepatic artery and portal vein blood supply, we studied the effect of curcumin- lipiodol emulsion and anti-adhesion film on the liver function and survival time of VX2 liver cancer rabbit and its effect on anti-angiogenesis in residual tumor.

\section{Methods}

\section{Animals and agents}

A total of 40 healthy New Zealand white rabbits, aged from four to five months and weighing from 2.0 to $2.5 \mathrm{~kg}$, were obtained from the Wushi Experimental Animal Center,
Fuzhou City, Fujian Province of China. Animal certificate number is 2007001103227. Curcumin and lipiodol were purchased from Xi'an Yuansen biological company. Curcumin was dissolved in dimethyl sulfoxide (DMSO). Absorbable anti-adhesion film (bai fei mi) was purchased from Beijing Bailikang biochemical company. CD34 and VEGF antibodies, Strept Avidin-Biotin Complex (SABC) kits, and Diaminobenzidine (DAB) kits were purchased from Wuhan Ph. D company.

\section{Preparing the VX2 tumor models in rabbits}

VX2 solid tumor cells were obtained from Shanghai Biosheng Bio-Company. The cell density was adjusted to $2 \times 10^{7}$ cells $/ \mathrm{mL}$, and $0.5 \mathrm{~mL}$ of VX2 tumor cells were inoculated into the lateral muscle of rabbit hind legs to construct VX2 hepatoma tumor-bearing rabbit model. Two weeks later the tumor can grow to $1-2 \mathrm{~cm}^{3}$. General anesthesia surgery was performed to obtain the tumor under aseptic conditions. Finally, the fish-like tissue with active tumor cells was cut into $1-2 \mathrm{~mm}^{3}$ tumor mass. Under anesthesia induced by Lumingning II and ketamine hydrochloride, a cut of about 3 to $4 \mathrm{~cm}$ is made at the bottom of the xiphoid process. Microsurgical tweezers were used to construct a deep fistula of about $5 \mathrm{~mm}$ in the left lateral lobe of the liver. Eye tweezers clip small tumor tissue into the fistula. After all the operations were completed, the rabbits who had undergone open surgery were irradiated with an infrared lamp for $30 \mathrm{~min}$. Infrared radiation promotes blood circulation and promotes tissue repair. Three days after operation the model rabbits were injected intramuscularly with $80 \mathrm{U}$ penicillin to prevent infection.

\section{Establishment of hepatic artery and portal vein ducts and establishment of in vitro indwelling model}

Two weeks later, the model rabbits were examined by computer tomography (CT) to confirm the successful planting of VX2 liver cancer. General anesthesia was induced and an incision was made to expose the liver and stomach. Gastroduodenal artery is carefully separated from surrounding tissue and will fully expose at least two centimeters. When it was sufficient, Epidural catheters were 

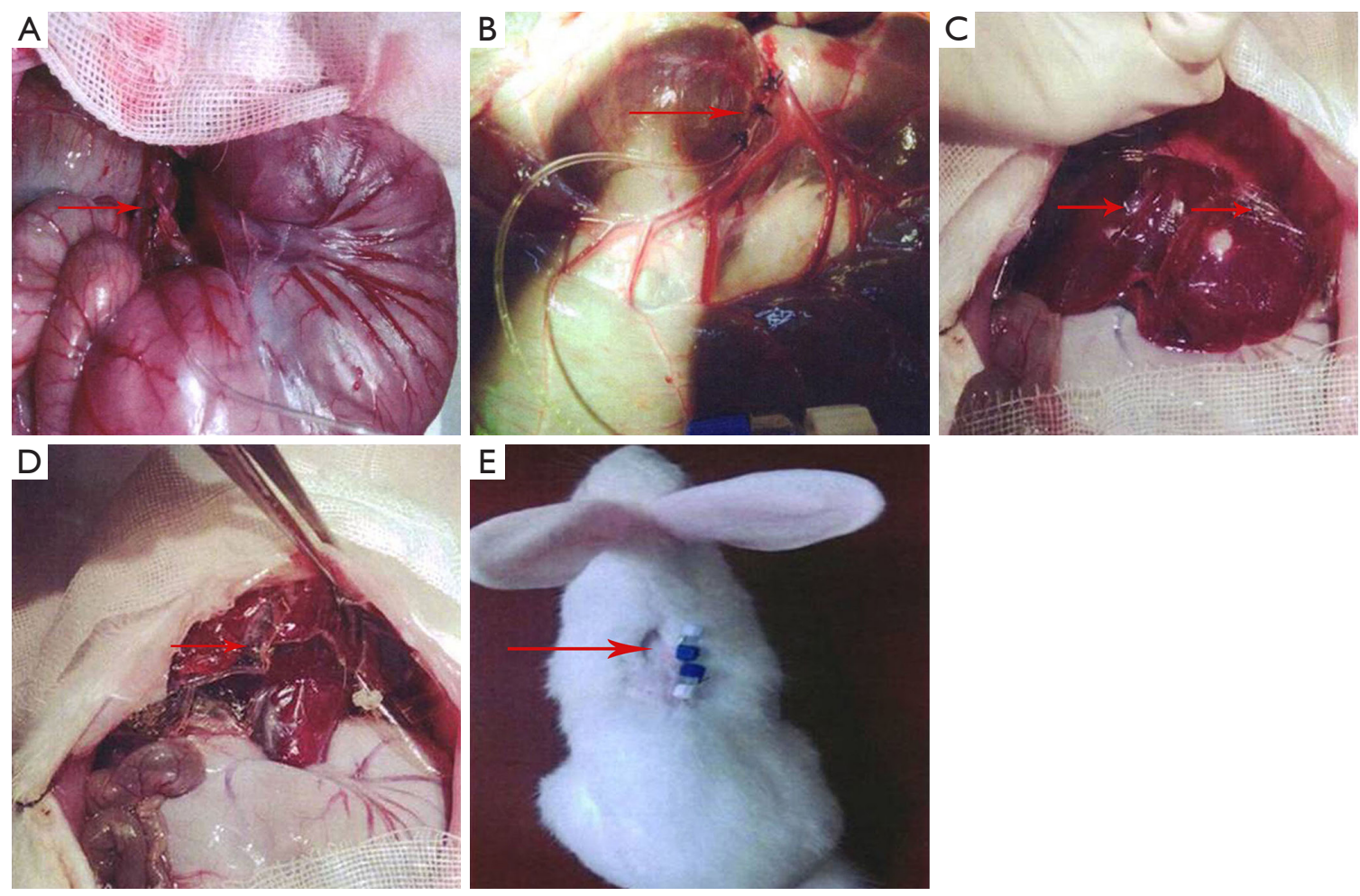

Figure 1 Two weeks later, the VX2 liver cancer rabbit model was successfully constructed. Next is the construction of the hepatic artery and portal vein catheterization model. (A) Establish the gastroduodenal artery intubation model (the arrow shows the gastroduodenal cannula); (B) establish the middle colic vein intubation model (arrows indicate cannulation of the middle colonic vein); (C) the anti-adhesion film wraps the diaphragmatic aspect of liver (the arrow shows the anti-adhesive membrane wrapped on the diaphragmatic aspect of liver); (D) the antiadhesion film wraps the facies inferior hepatis (the arrow shows the anti-adhesive membrane wrapped on the facies inferior hepatis); (E) the outer port of the catheter is fixed to the back of the rabbit (the arrow shows the outer orifice of the catheter).

inserted into the gastroduodenal artery to reach the proper hepatic artery as a substitute for administrating device in vitro (Figure 1). A methylene blue dye is then injected at the end of the catheter to confirm that the catheter has reached the proper hepatic artery. The same method was used to isolate the right branch of the middle colic vein (Figure 1). Epidural catheters were inserted into the right branch of the middle colic vein to reach the portal vein. Connect the syringe (containing saline) to the end of the catheter. If the dark red vein blood is seen to flow out, the model is successfully established. Flush the catheter with $1 \mathrm{ml}$ sodium heparin saline and screw on the cap on the outside of the catheter. After the successful construction of the indwelling catheter, the corresponding group should also wrap the anti-adhesion film around the liver (Figure 1). The outer opening of the catheter was sutured to the back of the model rabbit (Figure 1). Finally, the rabbit abdominal cavity was closed and routinely sterilized. After all the surgical procedures were completed, the homemade animal corsets were attached to the abdominal wounds of the model rabbits. The homemade corset is made of a bandage that mimics the medical abdomen belt. It is mainly used to stop bleeding and promote wound healing. Furthermore, anti-infection and nutritional support cannot be ignored.

\section{Experimental design}

After positioning the catheter within the proper hepatic artery and the portal vein, the embolic emulsion was injected into the catheter supplying the tumor and antiadhesion film was placed around the liver. Because each group of rabbits adopted different treatment strategies, the rabbits were divided into the following four groups. For the control group (group A), lipiodol emulsion $(0.2 \mathrm{~mL} / \mathrm{kg}$ ) was injected into the liver through two indwelling catheters in vitro. For the anti-adhesion film group (group B), Model 
rabbits not only need to be injected with lipiodol emulsion through the catheter, but also need to wrap the antiadhesion film around the liver. For the curcumin group (group C), a mixture of curcumin $(5 \mathrm{mg} / \mathrm{kg}$ ) and lipiodol $(0.2 \mathrm{~mL} / \mathrm{kg})$ was injected carefully into the liver through two indwelling catheters in vitro. For the combined treatment group (group D), rabbits need to be injected with both lipiodol $(0.2 \mathrm{~mL} / \mathrm{kg})$ and curcumin emulsion $(5 \mathrm{mg} / \mathrm{kg})$ through the catheter at the same time, and an anti-adhesion film needs to be wrapped around the liver.

\section{Detection of blood liver function before and after embolization}

Aspartate transaminase (AST) and alanine aminotransferase (ALT) are the most important enzymes in liver function tests, which are often used to assess the damage of liver cells (10). One day before embolization, the rabbits' marginal venous blood samples were collected to detect the changes of ALT and AST indexes. Then on the 3rd, 7th and 14th days after embolization, peripheral blood samples were collected for liver function testing. Finally, ALT and AST indexes were compared before and after embolization.

\section{Specimens}

After the successful construction of tumor-bearing rabbit VX2 liver cancer, one model rabbit was randomly selected from each group (not sacrificed). General anesthesia surgery was performed to open the abdominal cavity. A total of $1 \mathrm{~cm}^{3}$ of non-necrotic area around the tumor and surrounding normal liver tissue were excised. The specimen was soaked in $10 \%$ neutral formalin for 24 hours and embedded in paraffin. Tumor specimens were then stained by standard haematoxylin and eosin (H\&E) for histopathological diagnosis.

\section{Immunobistochemical staining}

Two weeks after embolization, one rabbit was randomly selected from each group (not sacrificed). Tumor tissue was obtained in the same manner as the preparation method of the pathological specimen. The tumor tissue was immersed in a formaldehyde solution for fixation, then embedded in paraffin, and finally sectioned to obtain a tissue section having a thickness of $4 \mu \mathrm{m}$. Rabbit polyclonal antibody to VEGF (Boster Biological Technology, Wuhan, China) was applied at a working dilution of 1:100. Rabbit polyclonal antibodies to CD34 (Boster Biological Technology, Wuhan, China) were also diluted in accordance with this method. The streptavidin-peroxidase (SP) method was used for immunohistochemical staining. The target cells showed a positive reaction under the action of 3,3'-diaminobenzidine (DAB substrate kit). Phosphate buffer saline (PBS) was used as a negative control for immunohistochemistry.

\section{Levels of VEGF in tumors}

When tumor cells display yellow or brown particles in the cytoplasm and/or cell membrane, they are considered to be VEGF positive cells. Each slice can be randomly selected five fields at $\times 400$ magnification of an optical microscope. Then, the HMIAS-2000 automatic color image analysis system was used to analyze and process the pictures of the dyed cells. The positive staining cells should be segmented and edited in the early stage of image analysis. The mean optic density (MOD) of each positive target can be calculated after obtaining satisfactory images.

\section{Microvessel density}

CD34 is the most sensitive marker of endothelial cells, so it is often used to assess microvascular density (MVD) in immunohistochemical staining. Dark brown color of endothelial cells showed positive staining for CD34. In this study, the Weidner method (11) was used for statistical analysis. Firstly, the blood vessels of the whole slice were observed under the microscope at a magnification of 100 times, and then the 5 regions with the most densely distributed microvessels were selected as the "hot spots" for counting at a magnification of 400 times. One point to note when counting microvessel density is that there should be statistics for a complete tubular structure. Yellowstained endothelial cells and cell clusters that do not have a complete tubular structure should also be counted. Because they are not directly connected to the surrounding capillaries or tumor cells, they should also be included in the statistics. Finally, the mean number of microvessels in the five field of view was calculated.

\section{Protein extraction and Western blotting assays}

After 2 weeks of embolization, one rabbit in each group was randomly selected to open the abdominal cavity to obtain tumor tissue for immunohistochemical staining. Meanwhile, $50 \mathrm{mg}$ tumor tissue was obtained by the 
same method for Western blot analysis. The tissue pieces were first washed twice with PBS and then cut to about 1-2 $\mathrm{mm}^{3}$ size. Single cell pellets can be obtained after digestion, centrifugation, washing, and resuspension. A mixture of Radio-Immunoprecipitation Assay (RIPA) and phenylmethanesulfonyl fluoride (PMSF) (1:100) lyses tumor cells to obtain a total protein extract mixture. Equal amounts of proteins were electrophoresed by SDSpolyacrylamide gel electrophoresis (PAGE) and transferred to polyvinylidene fluoride membranes. Finally, the optical density of protein was analyzed by ImageJ1.50i software.

\section{Overall survival (OS)}

After collecting all the observations, each group of rabbits continued to feed. The overall survival time of the remaining 36 rabbits from the time of embolization to death was recorded.

\section{Statistical analysis}

All the measured data were expressed as mean \pm standard deviation (mean $\pm \mathrm{SD}$ ). Two-way ANOVA was used for comparison between groups at each time point. The Student's t test was used for comparison of two values between multiple groups. The Kaplan-Meier method and the log-rank test were used for the data processing of the overall survival time. SPSS 19.0 software was used to analyze the data. $\mathrm{P}<0.05$ and $\mathrm{P}<0.01$ were used to express a statistically significant difference.

\section{Results}

\section{VX2 liver cancer formation and double embolic patbway} construction in rabbits

Two weeks after the construction of the rabbit VX2 liver tumor model, abdominal CT scan showed that 40 New Zealand white rabbits were successfully constructed with a tumor size of about 1 to $2 \mathrm{~cm}^{3}$. However, the structure of the hepatic artery and the portal vein intubation model resulted in the death of 4 experimental rabbits. One model rabbit died of massive blood loss due to portal vein injury. One model rabbit died of anesthesia sensitivity. One model rabbit died of massive hemorrhage due to rupture of the hepatic artery. One model rabbit died of postoperative intestinal obstruction. The remaining 36 VX2 liver cancer rabbits successfully completed all the observations and indicators of detection.

\section{Pathological findings}

Coagulative necrosis can be seen in the four groups of tumor specimens at low magnification of the microscope. The invasive growth of tumor cells was observed under high magnification, and the boundary of normal liver tissue was not clear. The thick wall vessels can be seen in the stroma of the tumor, which leads to the oppression of the hepatocytes and the narrowing of the hepatic sinusoids. The structure of normal hepatic sinusoids is rare. In addition, some liver cells showed obvious swelling. The spread of tumor cells can be seen in the normal liver tissues far away from the tumor tissue, and there are tumor nests or tumor suppositories (Figure 2).

\section{Changes in the level of AST and ALT}

There was no significant difference in AST and ALT between the four groups of rabbit venous blood 1 day before embolization $(\mathrm{P}>0.05)$. On the third day after embolization, the AST and ALT levels of the four groups of rabbits were higher than those 1 day before embolization. However, there was no significant difference between group B and group A $(\mathrm{P}>0.05)$. There was no statistical difference between group $\mathrm{C}$ and group $\mathrm{A}(\mathrm{P}>0.05)$, and the same was true for group $\mathrm{D}(\mathrm{P}>0.05)$. On the 7 th day after embolization, the AST and ALT levels of the four groups of venous blood were lower than the $3 \mathrm{rd}$ day. There was no significant difference between group B and group A $(\mathrm{P}>0.05)$. There was no difference between group $\mathrm{C}$ and group $\mathrm{A}(\mathrm{P}>0.05)$, and there was no difference in group $\mathrm{D}$ $(\mathrm{P}>0.05)$. On the 14th day after embolization, AST and ALT levels in the venous blood of the four groups of rabbits continued to decrease, but still higher than the AST and ALT levels at 1 day before embolization. However, there was no significant difference between group B and group $\mathrm{A}(\mathrm{P}>0.05)$. There was no difference between group $\mathrm{C}$ and group $\mathrm{A}(\mathrm{P}>0.05)$, and there was no difference in group $\mathrm{D}$ $(\mathrm{P}>0.05)$ (Tables 1,2; Figure 3).

\section{Effect of curcumin-lipiodol emulsion combined with anti- adbesion film on VEGF expression in tumor}

Immunohistochemical analysis demonstrated that the tumor tissues were strongly stained for VEGF in Group A. The mean optic density of VEGF in group B was lower than those in the group A, but there was no significant difference between the two groups $(\mathrm{P}>0.05)$. The levels of VEGF in group $\mathrm{C}$ and $\mathrm{D}$ were significantly lower than those in 


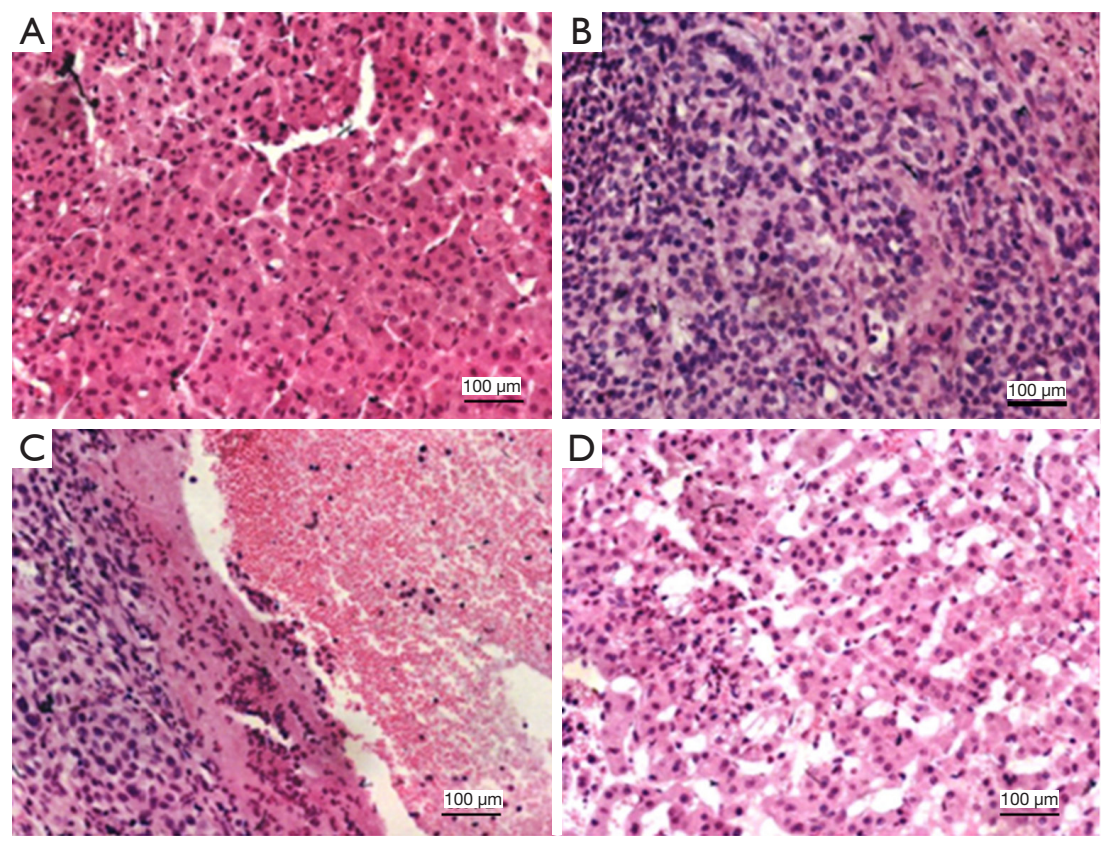

Figure 2 Tumour tissue 2 weeks after VX2 tumour implantation in a rabbit. The H-E staining results for the tumor specimens. (A,B) The thick wall vessels can be seen in the stroma of the tumor, which leads to the oppression of the hepatocytes and the narrowing of the hepatic sinusoids. (C) The spread of tumor cells can be seen in the normal liver tissues far away from the tumor tissue, and there are tumor nests or tumor suppositories. (D)The structure of normal hepatic sinusoids is rare. In addition, some liver cells showed obvious swelling. (original magnification $\times 200)$.

Table 1 The change of aspartate aminotransferase (AST) before and after embolization in each group $(\bar{x} \pm$ s)

\begin{tabular}{lccccc}
\hline \multirow{2}{*}{ Group } & $\mathrm{N}$ & \multicolumn{4}{c}{ AST $(\mathrm{U} / \mathrm{L})$} \\
\cline { 3 - 6 } & & 1 day before embolization & 3 day after embolization & 7day after embolization & 14 day after embolization \\
\hline A & 9 & $40.86 \pm 1.21$ & $372.35 \pm 2.18$ & $180.92 \pm 1.26$ & $97.75 \pm 2.36$ \\
B & 9 & $37.90 \pm 2.42$ & $366.48 \pm 1.63$ & $180.78 \pm 2.06$ & $99.63 \pm 1.68$ \\
C & 9 & $38.92 \pm 1.55$ & $371.10 \pm 0.78$ & $177.22 \pm 1.37$ & $100.53 \pm 0.97$ \\
D & 9 & $45.28 \pm 0.98$ & $365.49 \pm 1.52$ & $177.89 \pm 0.93$ & $97.50 \pm 1.38$ \\
\hline
\end{tabular}

At each time point, there was no statistical difference among the groups. There was no statistical difference between the change trends of each group.

Table 2 The change of alanine aminotransferase (ALT) before and after embolization in each group $(\bar{x} \pm$ s)

\begin{tabular}{lccccc}
\hline & & & \multicolumn{3}{c}{ ALT $(\mathrm{U} / \mathrm{L})$} \\
\cline { 3 - 6 } Group & $\mathrm{N}$ & 1 day before embolization & 3 day after embolization & 7day after embolization & 14 day after embolization \\
\hline A & 9 & $50.01 \pm 0.53$ & $260.91 \pm 1.36$ & $167.35 \pm 3.16$ & $90.25 \pm 1.36$ \\
B & 9 & $51.24 \pm 0.48$ & $252.51 \pm 2.01$ & $164.54 \pm 2.51$ & $91.80 \pm 1.56$ \\
C & 9 & $54.39 \pm 0.64$ & $253.43 \pm 1.84$ & $169.25 \pm 1.83$ & $88.65 \pm 1.37$ \\
D & 9 & $49.00 \pm 0.76$ & $258.75 \pm 2.27$ & $166.78 \pm 2.09$ & $88.45 \pm 1.09$ \\
\hline
\end{tabular}

At each time point, there was no statistical difference among the groups. There was no statistical difference between the change trends of each group. 

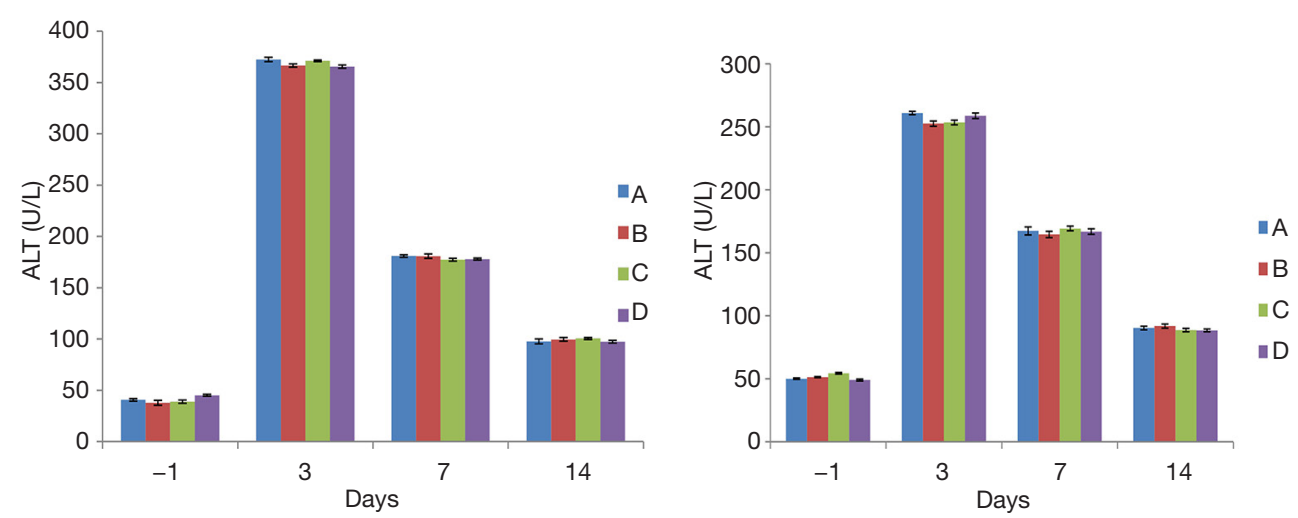

Figure 3 Hepatocyte damage of the different treatments on VX2 liver cancer in the rabbit. Rabbits with VX2 liver cancer were divided into four groups $(\mathrm{n}=10)$. Groups A, B, C and D were treated with lipiodol emulsion, anti-adhesion film, curcumin-lipiodol emulsion and curcumin-lipiodol emulsion + anti-adhesion film, respectively. (A) The alanine aminotransferase (AST) of rabbits in each group showed no difference at different time points; (B) the change trend of aspartate aminotransferase (ALT) is consistent with that of AST, but there is still no difference between each group at different time points.

the group $\mathrm{A}(\mathrm{P}<0.01)$. In addition, the decrease of VEGF in the group $\mathrm{D}$ was most obvious, and the group $\mathrm{D}$ had a significant difference compared with the group $\mathrm{C}(\mathrm{P}<0.05)$ (Figure 4).

\section{Effect of curcumin-lipiodol emulsion combined with anti- adbesion film on MVD}

Figure 5 shows typical CD34 immunohistochemical staining images and the effects of various treatments on MVD. These results indicate that curcumin-lipiodol emulsion decreased the MVD. In addition, the combination of curcumin-lipiodol emulsion and anti-adhesion film significantly decreased the MVD of the tumor tissue in group $\mathrm{D}$ compared with that in group $\mathrm{A}(\mathrm{P}<0.01)$. The decrease in group $\mathrm{D}$ was the most obvious, and the difference between group D and group $\mathrm{C}$ was also significant $(\mathrm{P}<0.05)$.

\section{The protein expression of VEGF in tumor}

Western blot analysis showed that the expression of VEGF was consistent with immunohistochemical staining. The mean relative protein expression of VEGF was $2.96 \pm 0.14$, $2.65 \pm 0.14,1.83 \pm 0.08$ and $1.61 \pm 0.07$ in the group A, group B, group $\mathrm{C}$ and group $\mathrm{D}$, respectively. The expression levels of VEGF protein in the combined curcumin-lipiodol emulsion and anti-adhesion film groups were the lowest compared with the other three groups. There was a significant difference between group $\mathrm{D}$ and group $\mathrm{A}(\mathrm{P}<0.01$; Figure 6). More importantly, there was also a significant difference between group $\mathrm{D}$ and group $\mathrm{C}(\mathrm{P}<0.05$; Figure 6).

\section{Survival time follow-up}

Curcumin has the effect of inhibiting tumor angiogenesis. However, curcumin does not significantly reduce the tumor volume in a short period of time, and it does not cure the tumor. Therefore, with the progress of liver cancer, the rabbits gradually died of liver cancer. The longest survival period is 40 days. Rabbits combined treatment with curcumin-lipiodol emulsion and anti-adhesion film (group D) had a significantly longer overall survival than those in the other three group. The OS time of VX2 liver cancer rabbits in group $\mathrm{D}$ was significantly different from group $\mathrm{A}(\mathrm{P}<0.01 ;$ Figure 7$)$. The OS time of group $\mathrm{C}$ VX2 liver cancer rabbits was also significantly different from that of group $\mathrm{A}(\mathrm{P}<0.01$; Figure 7$)$. In addition, there was also a significant difference between group $\mathrm{D}$ and group $\mathrm{C}$ $(\mathrm{P}<0.05$; Figure 7$)$.

\section{Discussion}

There are three main methods for constructing the rabbit model with VX2 liver cancer, of which the method of opening the abdominal cavity to inoculate the tumor has the highest success rate (12). The incidence of single tumor is high in this method, but the rate of ectopic implantation 

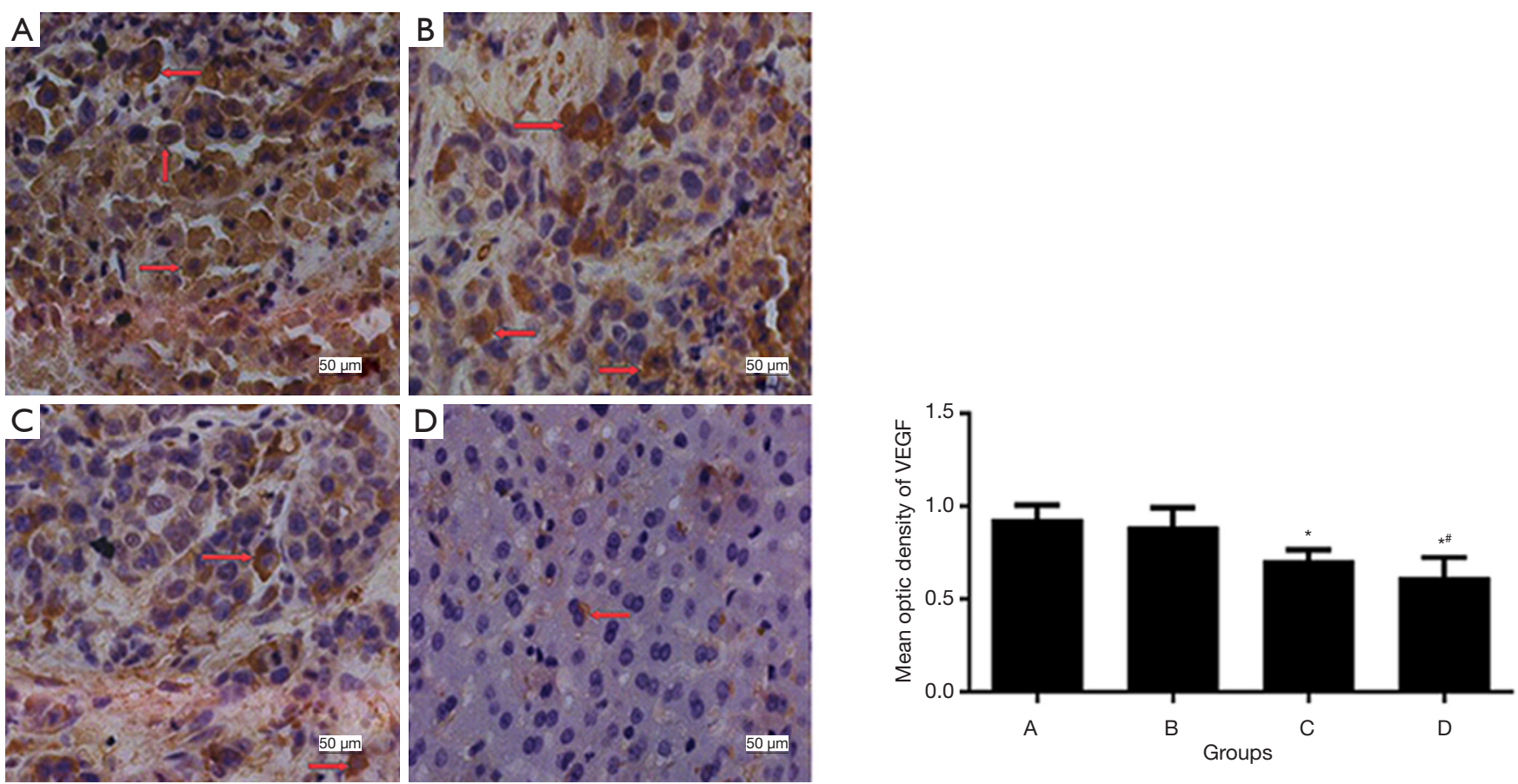

Figure 4 Immunohistochemical staining images of VEGF in tumor sections. Tissues were treated with (A) lipiodol emulsion, (B) lipiodol emulsion + anti-adhesion film, (C) curcumin-lipiodol emulsion and (D) curcumin-lipiodol emulsion + anti-adhesion film (n=9) (SABC, $\times 400)$. The VEGF expression in groups A, B, C and D was evaluated as strongly positive, positive, weakly positive and weakly positive, respectively. Red arrow indicates intracellular expression of VEGF. The mean optical density of VEGF expression levels in Groups D and C was significantly different from that in Group A. In addition, the group $\mathrm{D}$ had a significant difference compared with the group $\mathrm{C}$ ( $\mathrm{P}<0.05$ ) ${ }^{* *} \mathrm{P}<0.01$ and ${ }^{*} \mathrm{P}<0.05$ vs. group $\mathrm{A} .{ }^{*} \mathrm{P}<0.05$ vs. group $\left.\mathrm{C}\right)$. VEGF, vascular endothelial growth factor.

is very low. The surgical trauma caused by this open surgery method has almost no effect on the OS time. This method is also helpful in the construction of hepatic artery and portal vein intubation models. The normal liver tissue is a double blood supply, of which $75 \%$ of the blood is supplied by the portal vein and the other $20 \%$ is supplied by the hepatic artery (13). However, $95 \%$ of the blood perfusion of liver cancer is directly derived from the hepatic artery (14). Blood from the hepatic artery primarily supplies the central region of the tumor. Another 5\% of the blood perfusion comes from the portal vein, which supplies the marginal portion of the tumor. The study of the vascular cast of liver cancer showed that $75.6 \%$ of liver cancer nodules were derived from the branches of the portal vein. In liver cancer nodules less than $3 \mathrm{~cm}$ in diameter, $20 \%$ of cancerous nodules are only supplied by the portal vein alone. Therefore, the blockage of the portal vein also plays a very important role in the treatment and recurrence of liver cancer (15). Related research has confirmed that there was no significant difference in the degree of liver injury between the hepatic artery combined with portal vein embolization and TACE alone in the treatment of liver cancer, but the treatment effect was much better than that of TACE alone (16). As mentioned earlier, the longterm efficacy and survival rate of patients with liver cancer who are treated with TACE alone are not ideal (17). The main reason is the incomplete embolization of residual tumors and the formation of anoxic microenvironment after embolization. This microenvironment promotes massive angiogenesis in residual tumor tissue, ultimately accelerating tumor recurrence and metastasis. Angiogenesis is regulated by a range of angiogenic factor and angiogenesis inhibitors. Dynamic equilibrium can be reached between two cytokines under normal conditions. When the tumor occurs, the balance will be broken and the factors that promote angiogenesis will be synthesized and massively released. VEGF is the most important cytokine of all known angiogenic factors (18). The hypoxic microenvironment will be created in the tumor after embolization $(19,20)$. This micro environment can significantly stimulate the 

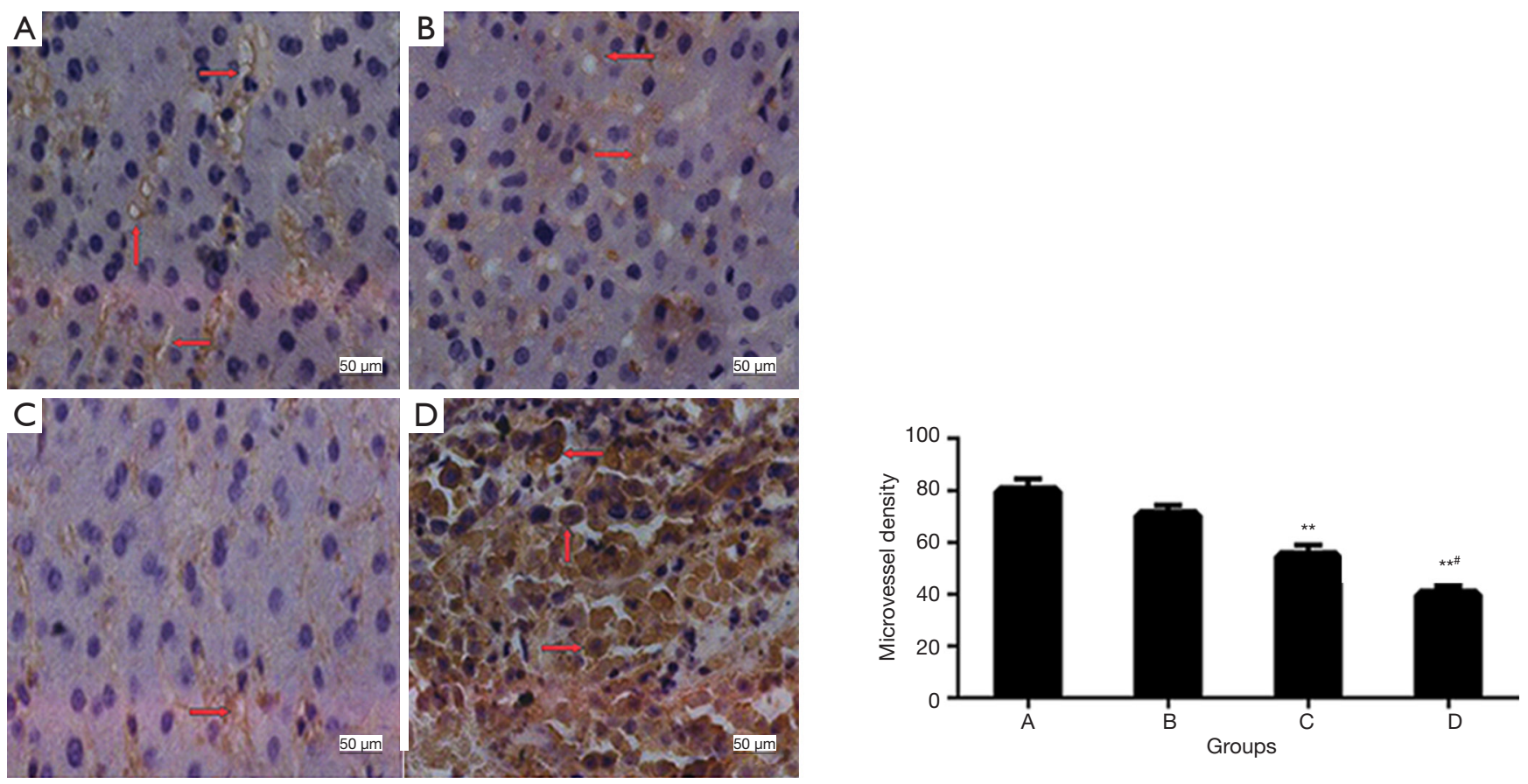

Figure 5 Hotspots of CD34 immunohistochemical staining and MVD in liver cancer tissues. Tissues were treated with (A) lipiodol emulsion; (B) lipiodol emulsion + anti-adhesion film; (C) curcumin-lipiodol emulsion and (D) curcumin-lipiodol emulsion + anti-adhesion film (n=9) (SABC, ×400). Red arrows indicate microvessels. The MVD of liver cancer tissues of group D and group C was all significantly different from that of group $\mathrm{A}$. In addition, there was also a significant difference between group $\mathrm{D}$ and group $\mathrm{C}$ (** $\mathrm{P}<0.01$ vs. group $\mathrm{A}$ and ${ }^{\#} \mathrm{P}<0.05$ vs. group C). MVD, microvessel density.
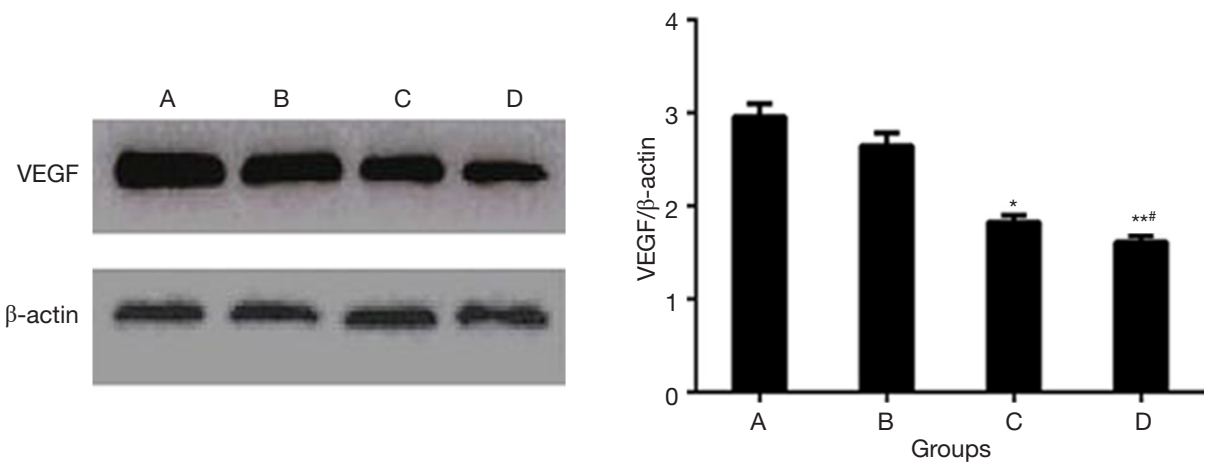

Figure 6 The relative expression of VEGF protein in each group was detected by Western blotting. There was a significant difference between group $\mathrm{D}$ and group $\mathrm{A}(\mathrm{P}<0.01)$. More importantly, there was also a significant difference between group $\mathrm{D}$ and group $\mathrm{C}(\mathrm{P}<0.05)$. $\left({ }^{*} \mathrm{P}<0.05\right.$ vs. group $\mathrm{A} ;{ }^{* \star} \mathrm{P}<0.01$ vs. group $\mathrm{A} ;{ }^{*} \mathrm{P}<0.05$ vs. group $\left.\mathrm{C}\right)$. VEGF, vascular endothelial growth factor.

expression of VEGF. VEGF can significantly promote the proliferation and migration of endothelial cells, resulting in a large number of neovascularization (21).

Curcumin is a plant antitoxin, which is different from the conventional chemotherapy drugs. It also has many effects, such as anti-inflammatory, anti-oxidation, antiatherosclerosis, anti-tumor and immune regulation (22). So, it gets more and more attention in the research of ovarian cancer, liver cancer and human Burkitt lymphoma. A growing number of studies have also shown that curcumin 

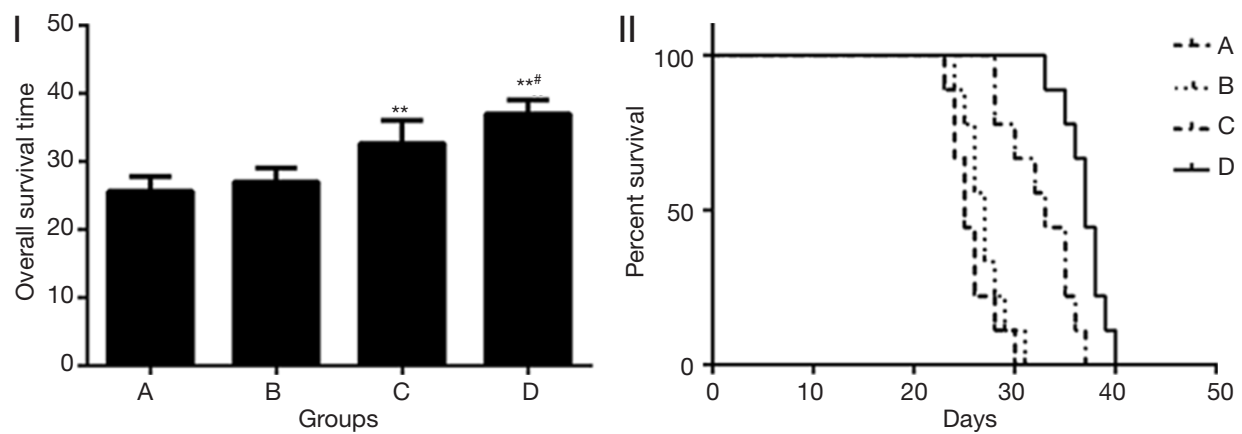

Figure 7 Analysis of the overall survival (OS) time in VX2 liver cancer rabbits. (I) The OS time of VX2 liver cancer rabbits in group D and $\mathrm{C}$ was all significantly different from group $\mathrm{A}$. In addition, there was also a significant difference between group $\mathrm{D}$ and group $\mathrm{C}\left({ }^{* *} \mathrm{P}<0.01\right.$ vs. group $\mathrm{A}$ and ${ }^{\#} \mathrm{P}<0.05$ vs. group C). (II) Kaplan-Meier survival curves of VX2 liver cancer rabbits with (A) lipiodol emulsion, (B) lipiodol emulsion + anti-adhesion film, (C) curcumin-lipiodol emulsion, and (D) curcumin-lipiodol emulsion + anti-adhesion film (n=9).

inhibits the proliferation of HCC cell lines such as HepG2, SMMC-7721, and BEL-7402ect (23-25). However, the latest research shows that the antitumor effect of curcumin is inseparable from its function of inhibiting tumor angiogenesis (26-28).

In this study, the treatment strategy of group D was to explore the anti-adhesion membrane combined with two-way infusion of curcumin-iodine emulsion to inhibit residual tumor angiogenesis. Also explore the impact of this method on animal survival. On the one hand, we analyzed the changes of serum ALT and AST before and after embolization in rabbit models. The results showed that the liver damage in the curcumin-lipiodol emulsion combined with anti-adhesion film group was very similar to the other three groups.

On the other hand, we explored the effects of curcuminlipiodol emulsion in combination with anti-adhesion membrane on angiogenesis. Two weeks after embolization, the levels of VEGF and MVD in the curcumin-lipiodol emulsion group were significantly lower than those in the simple lipiodol embolization group. The levels of VEGF and MVD in the curcumin-lipiodol emulsion combined with the anti-adhesion membrane group were also significantly lower than those in the simple lipiodol embolization group. The levels of VEGF and MVD in the combined adhesion membrane group were more significantly reduced. Therefore, we conclude that curcumin-lipiodol emulsion can significantly reduce the expression of VEGF and inhibit the formation of neovascularization of the tumor. The results of this paper are consistent with recent foreign studies (29,30). More importantly, our results also fully demonstrated that curcumin-lipiodol emulsion combined with anti-adhesion film can play a more obvious antiangiogenic effect. The consistent results of Western blotting and immunohistochemistry also verify this view on the other side. Finally, the results of the OS time showed that the curcumin-lipiodol emulsion combined with antiadhesion film group prolonged the survival time of the tumor rabbits. The reason is that the curcumin-lipiodol emulsion injected into the hepatic artery and portal vein can be released slowly in the tumor. The bioavailability of embolic agents can be improved, which can act on related angiogenesis pathways and play a significant role in inhibiting tumor angiogenesis. We believe that anti-adhesive membranes have biological barriers and time-differentiating effects. The absorbable anti-adhesion membrane can inhibit the adhesion between VX2 liver cancer and normal liver tissue and adjacent tissues and organs, thereby reducing the chance of collateral vessel formation. When the two methods are combined, curcumin-lipiodol emulsion and anti-adhesion film can promote each other, enhance each other, complement each other, and finally achieve a more ideal therapeutic effect.

\section{Conclusions}

In summary, this study showed that the hepatic artery and portal vein infusion of curcumin-lipiodol emulsion supplying with tumor can inhibit VX2 rabbit hepatic neovascularization and extend the OS time after embolization. However, curcumin-lipiodol emulsion combined with anti-adhesion film can effectively enhance the effect of antiangiogenesis and prolong the OS time of the animals after embolization. The current findings are 
important for further exploration of the potential use of curcumin alone or as part of a combination therapy for liver cancer.

\section{Acknowledgments}

Funding: This work was supported by grants from the Natural Science Foundation of Fujian Province (No. 2017J01164) and Foundation supporting the army of Science and Technology of Zhangzhou (ZZ2016KD06).

\section{Footnote}

Conflicts of Interest: All authors have completed the ICMJE uniform disclosure form (available at http://dx.doi. org/10.21037/tcr.2019.12.15). The authors have no conflicts of interest to declare.

Ethical Statement: The authors are accountable for all aspects of the work in ensuring that questions related to the accuracy or integrity of any part of the work are appropriately investigated and resolved. Experiments were performed in compliance with national or institutional guidelines for the care and use of animals.

Open Access Statement: This is an Open Access article distributed in accordance with the Creative Commons Attribution-NonCommercial-NoDerivs 4.0 International License (CC BY-NC-ND 4.0), which permits the noncommercial replication and distribution of the article with the strict proviso that no changes or edits are made and the original work is properly cited (including links to both the formal publication through the relevant DOI and the license). See: https://creativecommons.org/licenses/by-ncnd/4.0/.

\section{References}

1. Torre LA, Bray F, Siegel RL, et al. Global cancer statistics, 2012. CA Cancer J Clin 2015;65:87-108.

2. Giuliani J, Marzola M, Pansini G. Liver metastases from primary rectal cancer: a multidisciplinary reverse approach. J Gastrointest Cancer 2013;44:368-9.

3. Zhou DY, Qin J, Huang J, et al. Zoledronic acid inhibits infiltration of tumor-associated macrophages and angiogenesis following transcatheter arterial chemoembolization in rat hepatocellular carcinoma models. Oncol Lett 2017;14:4078-84.
4. Liu G, Xiang T, Wu QF, et al. Curcumin suppresses the proliferation of gastric cancer cells by downregulating H19. Oncol Lett 2016;12:5156-62.

5. Liu F, Gao S, Yang Y, et al. Curcumin induced autophagy anticancer effects on human lung adenocarcinoma cell line A549. Oncol Lett 2017;14:2775-82.

6. Taverna S, Giallombardo M, Pucci M, et al. Curcumin inhibits in vitro and in vivo chronic myelogenous leukemia cells growth: a possible role for exosomal disposal of miR21. Oncotarget 2015;6:21918-33.

7. Wei $Y, P u X, Z$ Zhao L. Preclinical studies for the combination of paclitaxel and curcumin in cancer therapy (Review). Oncol Rep 2017;37:3159-66.

8. Kunnumakkara AB, Bordoloi D, Harsha C, et al. Curcumin mediates anticancer effects by modulating multiple cell signaling pathways. Clin Sci (Lond) 2017;131:1781-99.

9. Wang JH, Wu LQ, Jing SF, et al. Clinical application of absorbable anti-adhesive film in tendon repair. Ophthalmology 2015;95:403-9.

10. Tolstrup JS, Grønbæk M, Tybjærghansen A, et al. Alcohol intake, alcohol dehydrogenase genotypes, and liver damage and disease in the Danish general population. Am J Gastroenterol 2009;104:2182-8.

11. Wang W, DA R, Wang M, et al. Expression of brainspecific angiogenesis inhibitor 1 is inversely correlated with pathological grade, angiogenesis and peritumoral brain edema in human astrocytomas. Oncol Lett 2013;5:1513-8.

12. Chen JH, Lin YC, Huang YS, et al. Induction of VX2 carcinoma in rabbit liver: comparison of two inoculation methods. Lab Anim 2004;38:79-84.

13. Shinozaki K, Ebert O, Woo SL. Treatment of multi-focal colorectal carcinoma metastatic to the liver of immunecompetent and syngeneic rats by hepatic artery infusion of oncolytic vesicular stomatitis virus. Int $\mathrm{J}$ Cancer 2005;114:659-64.

14. Naumann M, Bonsall R, Gupta R. Chemoembolization with Drug-Eluting Beads Complicated by Intrahepatic Biloma. Semin Intervent Radiol 2011;28:212-7.

15. Beppu T, Okabe H, Okabe K, et al. Multiple utilities of portal vein embolization for patients with hepatocellular carcinoma. J Clinical Oncology 2012;30:319-.

16. Guo WC, He XF, Li YH, et al. The effect of sequential transcatheter arterial chemoembolization (TACE) and portal venous embolizations (PVE) vs. TACE or PVE alone on rabbit VX2 liver carcinoma and on liver regeneration. Eur Rev Med Pharmacol Sci 2016;20:3186-93.

17. Choi SB, Kim KS, Park YN, et al. The Efficacy of 
Hepatic Resection after Neoadjuvant Transarterial Chemoembolization (TACE) and Radiation Therapy in Hepatocellular Carcinoma Greater Than $5 \mathrm{~cm}$ in Size. J Korean Med Sci 2009;24:242-7.

18. Holmes DI, Zachary I. The vascular endothelial growth factor (VEGF) family: angiogenic factors in health and disease. Genome Biol 2005;6:209.

19. Virmani S, Rhee TK, Ryu RK, et al. Comparison of hypoxia-inducible factor-1alpha expression before and after transcatheter arterial embolization in rabbit VX2 liver tumors. J Vasc Interv Radiol 2008;19:1483-9.

20. Pleguezuelo M, Marelli L, Misseri M, et al. TACE versus TAE as therapy for hepatocellular carcinoma. Expert Rev Anticancer Ther 2008;8:1623-41.

21. Iavarone M, Lampertico P, Iannuzzi F, et al. Increased expression of vascular endothelial growth factor in small hepatocellular carcinoma. J Viral Hepat 2007;14:133-9.

22. Sirohi VK, Popli P, Sankhwar P, et al. Curcumin exhibits anti-tumor effect and attenuates cellular migration via Slit-2 mediated down-regulation of SDF-1 and CXCR4 in endometrial adenocarcinoma cells. J Nutr Biochem 2017;44:60-70.

23. Chen Y, Zhong W, Chen B, et al. Effect of curcumin on vascular endothelial growth factor in hypoxic HepG2 cells via the insulin-like growth factor 1 receptor signaling pathway. Exp Ther Med 2018;15:2922-8.

Cite this article as: Yang C, Xu Z, Nie K, Liu J. Effect of curcumin-lipiodol emulsion combined with absorbable anti-adhesion film through double embolic pathway on angiogenesis and overall survival (OS) time of VX2 liver cancer rabbits. Transl Cancer Res 2020;9(2):742-753. doi: 10.21037/ tcr.2019.12.15
24. Zhang YJ, Xiang H, Liu JS, et al. Study on the mechanism of AMPK signaling pathway and its effect on apoptosis of human hepatocellular carcinoma SMMC-7721 cells by curcumin. Eur Rev Med Pharmacol Sci 2017;21:1144-50.

25. Cao H, Wang Y, He X, et al. Codelivery of sorafenib and curcumin by directed self-assembled nanoparticles enhances therapeutic effect on hepatocellular carcinoma. Mol Pharm 2015;12:922-31.

26. Sharma RA, Gescher AJ, Steward WP. Curcumin: the story so far. Eur J Cancer 2005;41:1955-68.

27. Singh S, Khar A. Biological effects of curcumin and its role in cancer chemoprevention and therapy. Anticancer Agents Med Chem 2006;6:259-70.

28. Das T, Sa G, Saha B, et al. Multifocal signal modulation therapy of cancer: ancient weapon, modern targets. Mol Cell Biochem 2010;336:85.

29. Fu Z, Chen X, Guan S, et al. Curcumin inhibits angiogenesis and improves defective hematopoiesis induced by tumor-derived VEGF in tumor model through modulating VEGF-VEGFR2 signaling pathway. Oncotarget 2015;6:19469-82.

30. Zhang Y, Cao H, Hu YY, et al. Inhibitory effect of curcumin on angiogenesis in ectopic endometrium of rats with experimental endometriosis. Int J Mol Med 2011;27:87-94. 